\title{
Multilinear Hölder-type inequalities on Lorentz sequence spaces
}

\author{
by \\ Daniel Carando (Buenos Aires), Verónica Dimant (Buenos Aires) \\ and Pablo Sevilla-Peris (Valencia)
}

\begin{abstract}
We establish Hölder-type inequalities for Lorentz sequence spaces and their duals. In order to achieve these and some related inequalities, we study diagonal multilinear forms in general sequence spaces, and obtain estimates for their norms. We also consider norms of multilinear forms in different Banach multilinear ideals.
\end{abstract}

1. Introduction. Given a sequence $\alpha \in \ell_{\infty}$, the generalized Hölder inequality states that, for $1 \leq p \leq n$, there exists a constant $C>0$ such that for all $x_{1}, \ldots, x_{n} \in \ell_{p}$,

$$
\left|\sum_{k=1}^{\infty} \alpha(k) x_{1}(k) \cdots x_{n}(k)\right| \leq C\left\|x_{1}\right\|_{\ell_{p}} \cdots\left\|x_{n}\right\|_{\ell_{p}} .
$$

On the other hand, if $n<p<\infty$, again Hölder's inequality implies that (1) holds if and only if $\alpha \in \ell_{p /(p-n)}$. Moreover, it can be shown that the best constant $C$ in (1) is in each case $\|\alpha\|_{\ell_{\infty}}$ and $\|\alpha\|_{\ell_{p /(p-n)}}$. A natural question now is if inequalities analogous to (1) can be found in other Banach sequence spaces (see below for definitions). More precisely, given a Banach sequence space $E$, under what conditions on $\alpha \in \ell_{\infty}$ does there exist $C>0$ such that for all $x_{1}, \ldots, x_{n} \in E$,

$$
\left|\sum_{k=1}^{\infty} \alpha(k) x_{1}(k) \cdots x_{n}(k)\right| \leq C\left\|x_{1}\right\|_{E} \cdots\left\|x_{n}\right\|_{E} ?
$$

Our aim in this paper is to analyze the situation when $E$ is a Lorentz space $d(w, p)$ or its dual $d(w, p)^{*}$. Our two main results are:

Theorem 1.1. Let $\alpha \in \ell_{\infty}$ and $E=d(w, p)$ with $1 \leq p<\infty$. Then:

(a) If $n \leq p$, then (2) holds if and only if $\alpha \in d(w, p / n)^{*}$.

2010 Mathematics Subject Classification: 46B45, 46A45, 46G25.

Key words and phrases: sequence spaces, Hölder inequality, multilinear mapping. 
(b) If $n>p$, then (2) holds if and only if $\alpha \in m_{\Psi}$, where $m_{\Psi}$ is the Marcinkiewicz space associated with $\Psi(N)=\left(\sum_{k=1}^{N} w(k)\right)^{n / p}$. If in addition $w$ is $n /(n-p)$-regular, then we can replace $m_{\Psi}$ by $\ell_{\infty}$.

The best constant is $\|\alpha\|_{d(w, p / n)^{*}}$ in case (a), and $\|\alpha\|_{m_{\Psi}}$ in case (b).

Theorem 1.2. Let $\alpha \in \ell_{\infty}$ and $E=d(w, p)^{*}$ with $1 \leq p<\infty$. Then:

(a) If $n^{\prime} \leq p$, then (2) holds if and only if $\alpha \in \ell_{\infty}$.

(b) If $n^{\prime}>p>1$, then (2) holds if and only if $\alpha \in d\left(w^{\frac{n^{\prime}}{n^{\prime}-p}}, \frac{p^{\prime}}{p^{\prime}-n}\right)$.

(c) If $p=1$, then (2) holds if and only if $\alpha \in d\left(w^{n}, 1\right)$.

The best constant in each case is the norm of $\alpha$ in the corresponding space.

Our approach is to study multilinear forms on the corresponding sequence spaces. Inequality (2) can be read as the continuity of the diagonal multilinear form on $E$ with coefficients $(\alpha(k))_{k}$. This way to look at Hölder inequalities is crucial to our proofs of Theorems 1.1 and 1.2. Moreover, it motivates an analogous question in a more general framework: if $\mathfrak{A}$ is a Banach ideal of multilinear mappings and $E$ is a Banach sequence space, under what conditions on $\alpha \in \ell_{\infty}$ does the diagonal multilinear form with coefficients $(\alpha(k))_{k}$ belong to $\mathfrak{A}\left({ }^{n} E\right)$ ? As a direct application of our results in this general framework, we consider nuclear and integral multilinear forms on Lorentz spaces and their duals.

The article is organized as follows. In Section 2 we introduce notation, definitions and some general results. Sections 3 and 4 are devoted to the proofs of Theorems 1.1 and 1.2. In Section 5 we broaden the object of our study, considering diagonal multilinear forms belonging to different ideals defined on general sequence spaces. Combining this with the results of the previous sections we characterize the diagonal integral (and nuclear) multilinear forms on Lorentz sequence spaces and their duals.

2. Preliminaries. Throughout the paper we use standard notation of Banach space theory. We consider complex Banach spaces $E, F, \ldots$ and their duals $E^{*}, F^{*}, \ldots$ Sequences of complex numbers are denoted by $x=$ $(x(k))_{k=1}^{\infty}$. By a Köthe sequence space (also known as Banach sequence space) we mean a Banach space $E \subseteq \mathbb{C}^{\mathbb{N}}$ such that $\ell_{1} \subseteq E \subseteq \ell_{\infty}$ and with the property that if $x \in \mathbb{C}^{\mathbb{N}}$ and $y \in E$ satisfy $|x(k)| \leq|y(k)|$ for all $k \in \mathbb{N}$ then $x \in E$ and $\|x\| \leq\|y\|$. For each element $x \in E$ in a Köthe sequence space its decreasing rearrangement $\left(x^{\star}(k)\right)_{k=1}^{\infty}$ is given by

$$
x^{\star}(k):=\inf \left\{\sup _{j \in \mathbb{N} \backslash J}|x(j)|: J \subseteq \mathbb{N}, \operatorname{card}(J)<k\right\} .
$$

A Köthe sequence space $E$ is called symmetric if $\left\|(x(k))_{k}\right\|_{E}=\left\|\left(x^{\star}(k)\right)_{k}\right\|_{E}$ for every $x \in E$. For each $N \in \mathbb{N}$ we consider the $N$-dimensional trun- 
cation $E_{N}:=\operatorname{span}\left\{e_{1}, \ldots, e_{N}\right\}$ (where $e_{n}$ denotes the $n$th canonical unit vector: $e_{n}(k)=\delta_{n, k}$ for all $k$ ) and we denote by $E_{0}$ the space of sequences in $E$ that are all 0 except for a finite number of coordinates. The canonical inclusion $i_{N}: E_{N} \hookrightarrow E$ and projection $\pi_{N}: E \rightarrow E_{N}$ are defined by $i_{N}\left((x(k))_{k=1}^{N}\right)=(x(1), \ldots, x(N), 0,0, \ldots)$ and $\pi_{N}\left((x(k))_{k=1}^{\infty}\right)=(x(k))_{k=1}^{N}$. Given two Banach spaces, we write $E=F$ if they are topologically isomorphic, and $E \stackrel{1}{=} F$ if they are isometrically isomorphic.

The Köthe dual of a Köthe sequence space $E$ is defined as

$$
E^{\times}:=\left\{z \in \mathbb{C}^{\mathbb{N}}: \sum_{j \in \mathbb{N}}|z(j) x(j)|<\infty \text { for all } x \in E\right\} .
$$

This can be considered even if $E$ is not normed. If $E$ is quasi-normed, $E^{\times}$ with the norm

$$
\|z\|_{E^{\times}}:=\sup _{\|x\|_{E} \leq 1} \sum_{j \in \mathbb{N}}|z(j) x(j)|
$$

is a Köthe sequence space. It is well known (see, for example, [3, Lemma $2.8])$ that $z \in E^{\times}$if and only if $\sum_{j \in \mathbb{N}} z(j) x(j)$ converges for all $x \in E$ and that

$$
\|z\|_{E^{\times}}=\sup _{\|x\|_{E} \leq 1}\left|\sum_{j \in \mathbb{N}} z(j) x(j)\right| .
$$

Also, $E^{\times}$is symmetric whenever $E$ is symmetric. Note that $\left(E_{N}\right)^{*} \stackrel{1}{=}\left(E^{\times}\right)_{N}$ for every $N$.

Following $[21,1 . d]$, a Köthe sequence space $E$ is said to be $r$-convex (with $1 \leq r<\infty$ ) if there exists a constant $\kappa>0$ such that for any choice $x_{1}, \ldots, x_{m} \in E$ we have

$$
\left\|\left(\left(\sum_{j=1}^{m}\left|x_{j}(k)\right|^{r}\right)^{1 / r}\right)_{k=1}^{\infty}\right\|_{E} \leq \kappa\left(\sum_{j=1}^{m}\left\|x_{j}\right\|_{E}^{r}\right)^{1 / r} .
$$

On the other hand, $E$ is $s$-concave (with $1 \leq s<\infty$ ) if there is a constant $\kappa>0$ such that

$$
\left(\sum_{j=1}^{m}\left\|x_{j}\right\|_{E}^{s}\right)^{1 / s} \leq \kappa\left\|\left(\left(\sum_{j=1}^{m}\left|x_{j}(k)\right|^{s}\right)^{1 / s}\right)_{k=1}^{\infty}\right\|_{E}
$$

for all $x_{1}, \ldots, x_{m} \in E$. We denote by $\mathbf{M}^{(r)}(E)$ and $\mathbf{M}_{(s)}(E)$ the smallest constants in the respective inequalities. Recall that $E$ is $r$-convex ( $s$ concave) if and only if $E^{*}$ is $r^{\prime}$-concave $\left(s^{\prime}\right.$-convex), where $r^{\prime}$ and $s^{\prime}$ are the conjugates of $r$ and $s$ respectively (see [21, 1.d.4]). Moreover, we have $M^{(r)}(E)=M_{\left(r^{\prime}\right)}\left(E^{*}\right)\left(M_{(s)}(E)=M^{\left(s^{\prime}\right)}\left(E^{*}\right)\right)$. If $E$ is $r$-convex for some $1<r<\infty$ or $s$-concave for some $1 \leq s<\infty$, then we say that $E$ has non-trivial convexity or non-trivial concavity. 
Following standard notation, given a symmetric Köthe sequence space $E$ we consider the fundamental function of $E$ :

$$
\lambda_{E}(N):=\left\|\sum_{k=1}^{N} e_{k}\right\|_{E}
$$

for $N \in \mathbb{N}$. For a detailed study and general facts on Köthe sequence spaces, see $[20,21,28,3,19]$.

REMARK 2.1. With this notation we can give a first positive answer to our question. If $E$ is $n$-concave, then $\alpha$ satisfies (2) if and only if $\alpha \in \ell_{\infty}$. Indeed, it is easily seen that being $n$-concave implies $E \hookrightarrow \ell_{n}$ (given $x \in E$, just take $x_{k}=x(k) e_{k} \in E$ and apply the definition of concavity). This and (1) immediately show that (2) holds for any $\alpha \in \ell_{\infty}$. Conversely, if (2) holds, considering $x_{1}=\cdots=x_{n}=e_{k}$ we obtain $|\alpha(k)| \leq C$ for all $k$, so $\alpha$ belongs to $\ell_{\infty}$.

The space of continuous linear operators between two Banach spaces $E, F$ will be denoted by $\mathcal{L}(E ; F)$, and the space of continuous $n$-linear mappings $E_{1} \times \cdots \times E_{n} \rightarrow F$ by $\mathcal{L}\left(E_{1}, \ldots, E_{n} ; F\right)$; with the norm

$$
\|T\|:=\sup \left\{\left\|T\left(x_{1}, \ldots, x_{n}\right)\right\|_{F}:\left\|x_{i}\right\|_{E_{i}} \leq 1, i=1, \ldots, n\right\},
$$

the latter is a Banach space. If $E_{1}=\cdots=E_{n}=E$ we write $\mathcal{L}\left({ }^{n} E ; F\right)$ and whenever $F=\mathbb{C}$ we simply write $\mathcal{L}\left(E_{1}, \ldots, E_{n}\right)$ or $\mathcal{L}\left({ }^{n} E\right)$.

A mapping $P: E \rightarrow F$ is a continuous $n$-homogeneous polynomial if there exists an $n$-linear mapping $T \in \mathcal{L}\left({ }^{n} E ; F\right)$ such that $P(x)=T(x, \ldots, x)$ for every $x \in E$. The space of all continuous $n$-homogeneous polynomials from $E$ to $F$ is denoted by $\mathcal{P}\left({ }^{n} E ; F\right)$; endowed with the norm $\|P\|=$ $\sup _{\|x\| \leq 1}\|P(x)\|$, it is a Banach space. If $P$ is an $n$-homogeneous polynomial and $T$ is the associated symmetric $n$-linear mapping, then the polarization formula gives (see [9, Proposition 1.8])

$$
\|P\| \leq\|T\| \leq \frac{n^{n}}{n !}\|P\| .
$$

A general study of polynomials on Banach spaces can be found in [9].

Ideals of multilinear forms were introduced in [23]. Let us recall the definition. An ideal of multilinear forms is a subclass $\mathfrak{A}$ of $\mathcal{L}$, the class of all multilinear forms, such that for any Banach spaces $E_{1}, \ldots, E_{n}$ the set

$$
\mathfrak{A}\left(E_{1}, \ldots, E_{n}\right)=\mathfrak{A} \cap \mathcal{L}\left(E_{1}, \ldots, E_{n}\right)
$$

satisfies:

- For any $\gamma_{1} \in E_{1}^{*}, \ldots, \gamma_{n} \in E_{n}^{*}$, the mapping

$$
\left(x_{1}, \ldots, x_{n}\right) \mapsto \gamma_{1}\left(x_{1}\right) \cdots \gamma_{n}\left(x_{n}\right)
$$

belongs to $\mathfrak{A}\left(E_{1}, \ldots, E_{n}\right)$.

- If $S, T \in \mathfrak{A}\left(E_{1}, \ldots, E_{n}\right)$, then $S+T \in \mathfrak{A}\left(E_{1}, \ldots, E_{n}\right)$. 
- If $T \in \mathfrak{A}\left(E_{1}, \ldots, E_{n}\right)$ and $S_{i} \in \mathcal{L}\left(F_{i}, E_{i}\right)$ for $i=1, \ldots, n$, then $T \circ$ $\left(S_{1}, \ldots, S_{n}\right) \in \mathfrak{A}\left(F_{1}, \ldots, F_{n}\right)$.

An ideal of multilinear forms is called normed if for each $E_{1}, \ldots, E_{n}$ there is a norm $\|\cdot\|_{\mathfrak{A}\left(E_{1}, \ldots, E_{n}\right)}$ in $\mathfrak{A}\left(E_{1}, \ldots, E_{n}\right)$ such that

- $\left\|\left(x_{1}, \ldots, x_{n}\right) \mapsto \gamma_{1}\left(x_{1}\right) \cdots \gamma_{n}\left(x_{n}\right)\right\|_{\mathfrak{A}\left(E_{1}, \ldots, E_{n}\right)}=\left\|\gamma_{1}\right\| \cdots\left\|\gamma_{n}\right\|$.

- $\left\|T \circ\left(S_{1}, \ldots, S_{n}\right)\right\|_{\mathfrak{A}\left(F_{1}, \ldots, F_{n}\right)} \leq\|T\|_{\mathfrak{A}\left(E_{1}, \ldots, E_{n}\right)} \cdot\left\|S_{1}\right\| \cdots\left\|S_{n}\right\|$.

Analogously ideals of homogeneous polynomials were defined and studied in [10-13]. However, [12] shows that a polynomial is in a normed ideal of polynomials if and only if its associated multilinear mapping is in some ideal of multilinear forms. Hence, dealing with one or the other type of ideals will not lead to essentially different conclusions.

If $(a(k))_{k}$ and $(b(k))_{k}$ are real sequences, we write $a(k) \prec b(k)$ when there exists $C>0$ such that $a(k) \leq C b(k)$ for all $k \in \mathbb{N}$. Also, we write $a(k) \asymp b(k)$ when $a(k) \prec b(k)$ and $b(k) \prec a(k)$.

3. Lorentz spaces. Our aim in this section is to prove Theorem 1.1. Let us first recall the definition of Lorentz spaces; further details and properties can be found in [20, Section 4.e] and [21, Section 2.a]. Let $(w(k))_{k=1}^{\infty}$ be a decreasing sequence of positive numbers such that $w(1)=1, \lim _{k} w(k)=0$ and $\sum_{k=1}^{\infty} w(k)=\infty$ and let $1 \leq p<\infty$. Then the corresponding Lorentz sequence space, denoted by $d(w, p)$, is defined as the space of all sequences $(x(k))_{k}$ such that

$$
\|x\|=\sup _{\pi \in \Sigma_{\mathbb{N}}}\left(\sum_{k=1}^{\infty}|x(\pi(k))|^{p} w(k)\right)^{1 / p}=\left(\sum_{k=1}^{\infty}\left|x^{\star}(k)\right|^{p} w(k)\right)^{1 / p}<\infty
$$

where $\Sigma_{\mathbb{N}}$ denotes the group of permutations of the natural numbers. Each $d(w, p)$ is clearly a symmetric Köthe sequence space.

The sequence $w$ is said to be $\alpha$-regular $(0<\alpha<\infty)$ if $w(k)^{\alpha} \asymp$ $k^{-1} \sum_{j=1}^{k} w(j)^{\alpha}$, and regular if it is $\alpha$-regular for some $\alpha$.

In [24] it is proved that $d(w, p)$ is $r$-convex (and $\mathbf{M}^{(r)}(d(w, p))=1$ ) whenever $1 \leq r \leq p$. Also [24, Theorem 2] shows that, for $p<s<\infty$, $d(w, p)$ is $s$-concave if and only if $w$ is $s /(s-p)$-regular. It is non-trivially concave if and only if $w$ is 1-regular.

In [15] and [20] a description of $d(w, p)^{*}$, the dual of $d(w, p)$, is given as the space of those sequences $x$ such that there exists a decreasing $y \in B_{\ell_{p^{\prime}}}$ with

$$
\sup _{N} \frac{\sum_{k=1}^{N} x^{\star}(k)}{\sum_{k=1}^{N} y(k) w(k)^{1 / p}}<\infty
$$

for $p>1$. The norm in $d(w, p)^{*}$ is the infimum of the expressions above over 
all possible decreasing $y \in B_{\ell_{p^{\prime}}}$. For $p=1$,

$$
d(w, 1)^{*}=\left\{x:\|x\|=\sup _{N} \frac{\sum_{k=1}^{N} x^{\star}(k)}{\sum_{k=1}^{N} w(k)}<\infty\right\} .
$$

If $w$ is regular, an easier description of $d(w, p)^{*}$ with $p>1$ can be given (see [2] and [25]):

$$
d(w, p)^{*}=\left\{x:\left(\frac{x^{\star}(k)}{w(k)^{1 / p}}\right)_{k=1}^{\infty} \in \ell_{p^{\prime}}\right\} .
$$

The $\ell_{p^{\prime}}$ norm of this sequence is a positive homogeneous function of $x$ which, although not a norm, is equivalent to the norm in $d(w, p)^{*}$ (see $[25$, Theorem 1]).

The Lorentz spaces $d(w, p)$ are reflexive whenever $p>1$ [20, Section 4.e]. If $p=1$ the predual of $d(w, 1)$ can be described as (see $[26,14]$ )

$$
d_{*}(w, 1)=\left\{x \in c_{0}: \lim _{N \rightarrow \infty} \frac{\sum_{k=1}^{N} x^{\star}(k)}{\sum_{k=1}^{N} w(k)}=0\right\}
$$

with the norm

$$
\|x\|=\sup _{N} \frac{\sum_{k=1}^{N} x^{\star}(k)}{\sum_{k=1}^{N} w(k)} .
$$

Let us recall that, given a strictly positive, increasing sequence $\Psi$ such that $\Psi(0)=0$, the associated Marcinkiewicz sequence space $m_{\Psi}$ (see $[17$, Definition 4.1], also [7, 16]) consists of all sequences $(x(k))_{k}$ such that

$$
\|x\|_{m_{\Psi}}=\sup _{N} \frac{\sum_{k=1}^{N} x^{\star}(k)}{\Psi(N)}<\infty .
$$

In order to prove part (a) of Theorem 1.1 we make use of a general result. Let us recall first that if $E$ is a symmetric Köthe sequence space, its $n$-concavification $E_{(n)}$ (see [21, Section 1.d]) is defined as the set of those sequences $(z(k))_{k}$ such that $\left(|z(k)|^{1 / n}\right)_{k} \in E$. On $E_{(n)}$ we can define a symmetric quasi-norm by $\|z\|_{E_{(n)}}=\left\|\left(|z(k)|^{1 / n}\right)_{k}\right\|_{E}^{n}$. This quasi-norm satisfies the "monotonicity condition": if $z \in \mathbb{C}^{\mathbb{N}}$ and $w \in E_{(n)}$ are such that $|z(k)| \leq|w(k)|$ for all $k \in \mathbb{N}$ then $z \in E_{(n)}$ and $\|z\|_{E_{(n)}} \leq\|w\|_{E_{(n)}}$. If $E$ is $n$-convex and $\mathbf{M}^{(n)}(E)=1$, then $\|\cdot\|_{E_{(n)}}$ is actually a norm and $E_{(n)}$ turns out to be a symmetric Köthe sequence space.

We can now give the result we need. This could be deduced from a result on orthogonally additive polynomials on Banach lattices given in [4, Theorem 2.3]. However, in our setting (symmetric Köthe sequence spaces) it is easier to give a direct proof. Note that the Köthe dual is by definition the set in which we have some Hölder inequality. In (2) we aim at an $n$-linear Hölder inequality; it is no surprise then that the Köthe dual of $E_{(n)}$ appears. 
Lemma 3.1. Let $\alpha \in \ell_{\infty}$ and $E$ be a symmetric Köthe sequence space. Then (2) holds if and only if $\alpha \in\left(E_{(n)}\right)^{\times}$and the best constant in (2) is $\|\alpha\|_{\left(E_{(n)}\right)} \times$.

Proof. Let $\alpha$ satisfy (2); then there exists $C>0$ such that for every $x \in E$,

$$
\left|\sum_{k=1}^{\infty} \alpha(k) x(k)^{n}\right| \leq C\|x\|_{E}^{n} .
$$

This implies that $\sum_{k} \alpha(k) z(k)$ is finite for every $z \in E_{(n)}$, hence $\alpha \in\left(E_{(n)}\right)^{\times}$ and $\|\alpha\|_{\left(E_{(n)}\right) \times} \leq C$.

On the other hand, if $\alpha \in\left(E_{(n)}\right)^{\times}$take $x_{1}, \ldots, x_{n} \in E$. Note first that the inequality

$$
\left(\left|x_{1}(k)\right| \cdots\left|x_{n}(k)\right|\right)^{1 / n} \leq \frac{\left|x_{1}(k)\right|+\cdots+\left|x_{n}(k)\right|}{n}
$$

implies that $\left(\left(x_{1}(k) \cdots x_{n}(k)\right)^{1 / n}\right)_{k} \in E$ and so $z:=\left(x_{1}(k) \cdots x_{n}(k)\right)_{k} \in E_{(n)}$. As a consequence of (4) we have $\|z\|_{E_{(n)}} \leq\left\|x_{1}\right\|_{E} \cdots\left\|x_{n}\right\|_{E}$. Indeed, by dividing by $\left\|x_{1}\right\|_{E} \cdots\left\|x_{n}\right\|_{E}$, it is enough to prove the inequality for $\left\|x_{1}\right\|_{E}=$ $\cdots=\left\|x_{n}\right\|_{E}=1$. In this case we have

$$
\begin{aligned}
\|z\|_{E_{(n)}} & =\left\|\left(\left(\left|x_{1}(k) \cdots x_{n}(k)\right|\right)^{1 / n}\right)_{k}\right\|_{E}^{n} \leq\left\|\left(\frac{\left|x_{1}(k)\right|+\cdots+\left|x_{n}(k)\right|}{n}\right)_{k}\right\|_{E}^{n} \\
& \leq\left(\frac{\left\|x_{1}\right\|_{E}+\cdots+\left\|x_{n}\right\|_{E}}{n}\right)^{n}=1 .
\end{aligned}
$$

Therefore

$$
\begin{aligned}
\left|\sum_{k=1}^{N} \alpha(k) x_{1}(k) \cdots x_{n}(k)\right| & =\left|\sum_{k=1}^{N} \alpha(k) z(k)\right| \leq\|\alpha\|_{\left(E_{(n)}\right)}\|z\|_{E_{(n)}} \\
& \leq\|\alpha\|_{\left(E_{(n)}\right)} \times\left\|x_{1}\right\|_{E} \cdots\left\|x_{n}\right\|_{E}
\end{aligned}
$$

for every $N$. Thus (2) holds with $C=\|\alpha\|_{\left(E_{(n)}\right)}$, and this completes the proof.

The last inequality in the previous proof can be seen as an estimate of the norm of a multilinear form. Let us say that a multilinear form $T$ on a sequence space $E$ is called diagonal if there exists a sequence $\alpha$ such that for all $x_{1}, \ldots, x_{n} \in E$,

$$
T\left(x_{1}, \ldots, x_{n}\right)=\sum_{k=1}^{\infty} \alpha(k) x_{1}(k) \cdots x_{n}(k) .
$$

In this case we write $T=T_{\alpha}$. With this terminology, Lemma 3.1 states that diagonal $n$-linear forms on $E$ correspond to sequences $\alpha \in\left(E_{(n)}\right)^{\times}$and

$$
\left\|T_{\alpha}\right\|=\|\alpha\|_{\left(E_{(n)}\right)^{\times}} .
$$


The $n$-homogeneous polynomial associated to $T_{\alpha}$ is also called diagonal and is denoted $P_{\alpha}$.

REMARK 3.2. We observe in (3) the general relationship between the norms of a polynomial and its associated symmetric $n$-linear form. For diagonal forms and polynomials defined on a symmetric Köthe sequence space $E$ the situation is different. It is proved in the previous lemma that if $x_{1}, \ldots, x_{n}$ are in $E$ then $\left(\left(x_{1}(k) \cdots x_{n}(k)\right)^{1 / n}\right)_{k}$ also belongs to $E$ and

$$
\left\|\left(\left(x_{1}(k) \cdots x_{n}(k)\right)^{1 / n}\right)_{k}\right\|^{n} \leq\left\|x_{1}\right\| \cdots\left\|x_{n}\right\| .
$$

Thus, the norm of any multilinear diagonal form on $E$ coincides with the norm of its associated diagonal polynomial, that is, $\left\|T_{\alpha}\right\|=\left\|P_{\alpha}\right\|$.

Lemma 3.1 provides an abstract characterization of the sequences $\alpha$ such that inequality (2) is satisfied. However, the Köthe dual of the $n$ concavification of $E$ is not always the simplest way to obtain an explicit description of such sequences. Therefore, in some cases we use different approaches.

Now we prove our first theorem.

Proof of Theorem 1.1. For statement (a), since $n \leq p$, the $n$-concavification of $d(w, p)$ is the space $d(w, p / n)$. Then Lemma 3.1 gives the conclusion.

For (b), let $\alpha$ and $C>0$ satisfy (2) with $E=d(w, p)$. For any fixed $N \in \mathbb{N}$, let $J_{N} \subseteq \mathbb{N}$ be such that $\left|J_{N}\right|=N$. Then for any $\left(\lambda_{k}\right)_{k \in J_{N}} \subset \mathbb{C}$ with $\left|\lambda_{k}\right|=1$,

$$
\left|\sum_{k \in J_{N}} \alpha(k) \lambda_{k}^{n}\right| \leq C\left\|\sum_{k \in J_{N}} \lambda_{k} e_{k}\right\|_{d(w, p)}^{n}=C\left(\sum_{k=1}^{N} w(k)\right)^{n / p} .
$$

Choosing $\lambda_{k}$ and $J_{N}$ so that $\sum_{k \in J_{N}} \lambda_{k}^{n} \alpha(k)=\sum_{k=1}^{N} \alpha^{\star}(k)$ we get, for any $N$,

$$
\frac{\sum_{k=1}^{N} \alpha^{\star}(k)}{\left(\sum_{k=1}^{N} w(k)\right)^{n / p}} \leq C .
$$

Thus, $\alpha \in m_{\Psi}$ with $\Psi(N)=\left(\sum_{k=1}^{N} w(k)\right)^{n / p}$.

For the reverse inclusion, let $\alpha \in m_{\Psi}$. Without loss of generality we can assume $\alpha=\alpha^{\star}$. Consider the diagonal $n$-linear mapping $T_{\alpha}: d(w, p) \times \cdots \times$ $d(w, p) \rightarrow \mathbb{C}$. By Remark 3.2, $T_{\alpha}$ is continuous if and only if the associated polynomial $P_{\alpha}: d(w, p) \rightarrow \mathbb{C}$ is continuous, and their norms are equal. First of all,

$$
\left|P_{\alpha}(x)\right|=\left|\sum_{k=1}^{\infty} \alpha(k) x(k)^{n}\right| \leq \sum_{k=1}^{\infty} \alpha(k) x^{\star}(k)^{n} .
$$


If we prove that

$$
\sum_{k=1}^{N} \alpha(k) x^{\star}(k)^{n} \leq\|\alpha\|_{m_{\Psi}}\left(\sum_{k=1}^{N} w(k) x^{\star}(k)^{p}\right)^{n / p}
$$

for every $N$, then $\left|P_{\alpha}(x)\right| \leq\|\alpha\|_{m_{\Psi}}\|x\|_{d(w, p)}^{n}$ and the result will follow.

We can assume that $x=x^{\star}$. By the definition of $m_{\Psi}$ we have

$$
\begin{aligned}
\sum_{k=1}^{N} \alpha(k) x(k)^{n} & =\sum_{i=1}^{N-1}\left(\sum_{k=1}^{i} \alpha(k)\right)\left(x(i)^{n}-x(i+1)^{n}\right)+\left(\sum_{k=1}^{N} \alpha(k)\right) x(N)^{n} \\
& \leq\|\alpha\|_{m_{\Psi}} \sum_{i=1}^{N} \Psi(i)\left(x(i)^{n}-x(i+1)^{n}\right)+\|\alpha\|_{m_{\Psi}} \Psi(N) x(N)^{n} \\
& =\|\alpha\|_{m_{\Psi}}\left[\Psi(1) x(1)^{n}+\sum_{i=2}^{N}(\Psi(i)-\Psi(i-1)) x(i)^{n}\right] .
\end{aligned}
$$

To obtain (5), we need to prove that for every $N$,

$$
\Psi(1) x(1)^{n}+\sum_{i=2}^{N}(\Psi(i)-\Psi(i-1)) x(i)^{n} \leq\left(\sum_{k=1}^{N} w(k) x(k)^{p}\right)^{n / p} .
$$

We proceed by induction. For $N=1$, the inequality is obvious. By the induction hypothesis we have

$$
\begin{aligned}
\Psi(1) x(1)^{n}+ & \sum_{i=2}^{N+1}(\Psi(i)-\Psi(i-1)) x(i)^{n} \\
& \leq\left(\sum_{k=1}^{N} w(k) x(k)^{p}\right)^{n / p}+(\Psi(N+1)-\Psi(N)) x(N+1)^{n} .
\end{aligned}
$$

We want to show that the last expression is at most $\left(\sum_{k=1}^{N+1} w(k) x(k)^{p}\right)^{n / p}$. Equivalently, we have to prove

$$
\begin{aligned}
& \Psi(N+1)-\Psi(N) \\
& \quad \leq\left(\sum_{k=1}^{N+1} w(k)\left(\frac{x(k)}{x(N+1)}\right)^{p}\right)^{n / p}-\left(\sum_{k=1}^{N} w(k)\left(\frac{x(k)}{x(N+1)}\right)^{p}\right)^{n / p} .
\end{aligned}
$$

Consider the increasing function $\phi(t)=(t+w(N+1))^{n / p}-t^{n / p}$ (recall that $n \geq p)$. Since $x$ is decreasing, $\sum_{k=1}^{N} w(k) \leq \sum_{k=1}^{N} w(k)(x(k) / x(N+1))^{p}$. Hence

$$
\phi\left(\sum_{k=1}^{N} w(k)\right) \leq \phi\left(\sum_{k=1}^{N} w(k)\left(\frac{x(k)}{x(N+1)}\right)^{p}\right) ;
$$


but this is exactly what we want in (7). This gives (6), hence (5) holds and the result follows.

If in addition $w$ is $n /(n-p)$-regular, then it is easy to see that $m_{\Psi}$ is isomorphic to $\ell_{\infty}$. This completes the proof.

REMARK 3.3. It is known (and can be deduced, for example, from [16, Lemma 3.3]) that $m_{\Psi}$ is isomorphic to the dual of a Lorentz space $d(\bar{w}, 1)$ for some sequence $\bar{w}$, understanding $d(\bar{w}, 1)=\ell_{1}$ if $\bar{w}$ is not a null sequence.

In some cases, the sequence $\bar{w}$ can be determined. For example, for $\breve{w}(k)=\Psi(k)-\Psi(k-1)$, we have

$$
\frac{\sum_{k=1}^{N} \alpha^{\star}(k)}{\Psi(N)}=\frac{\sum_{k=1}^{N} \alpha^{\star}(k)}{\sum_{k=1}^{N} \breve{w}(k)} .
$$

If $\breve{w}$ is decreasing, we deduce that (2) holds for $E=d(w, p)$ if and only if $\alpha \in d(\breve{w}, 1)^{*}$. Moreover, there are universal constants $A_{n}, B_{n}$ (not depending on $\alpha)$ such that the best $C>0$ in (2) satisfies $A_{n}\|\alpha\|_{d(\breve{w}, 1)^{*}} \leq C \leq$ $B_{n}\|\alpha\|_{d(\breve{w}, 1)^{*}}$.

If $w$ is regular (i.e., 1-regular) and the sequence $\tilde{w}(k)=(k w(k))^{n / p} / k$ is decreasing we get another description, namely $m_{\Psi}=d(\tilde{w}, 1)^{*}$. Indeed, by the mean value theorem

$$
\Psi(k)-\Psi(k-1)=\frac{n}{p} z(k)^{n / p-1} w(k)
$$

for some $\sum_{j=1}^{k-1} w(j) \leq z(k) \leq \sum_{j=1}^{k} w(j)$. But $\sum_{j=1}^{k} w(j) \asymp k w(k)$ and $\sum_{j=1}^{k-1} w(j) \asymp(k-1) w(k-1) \geq(k-1) w(k) \succ k w(k)$. So we have $z(k) \asymp$ $k w(k)$. Consequently, $\breve{w}(k) \asymp(k w(k))^{n / p-1} w(k)=\tilde{w}(k)$, and since $(\tilde{w}(k))_{k}$ is decreasing, we have $m_{\Psi}=d(\tilde{w}, 1)^{*}$. Hence, in this case, (2) holds if and only if $\alpha \in d(\tilde{w}, 1)^{*}$.

Note that $\breve{w}(k) \asymp \tilde{w}(k)$ if and only if $w$ is regular. Also, if either $\breve{w}$ or $\tilde{w}$ is decreasing but does not converge to zero, then the corresponding Lorentz space $d(\cdot, 1)$ is in fact $\ell_{1}$ and then its dual is $\ell_{\infty}$.

In the following example we apply our results to the Lorentz sequence spaces $\ell_{p, q}$. For the particular case $q<n<p$, this example shows that the regularity condition in part (b) of Theorem 1.1 is sharp: for any $r<n /(n-p)$ there are $r$-regular sequences $w$ such that (2) does not hold for some $\alpha \in \ell_{\infty}$ and $E=d(w, p)$.

EXAMPLE 3.4. Special cases of Lorentz sequence spaces are the $\ell_{p, q}$ spaces. For $p>q \geq 1$ they are defined as

$$
\ell_{p, q}=\left\{x:\|x\|=\left(\sum_{k=1}^{\infty} \frac{x^{\star}(k)^{q}}{k^{1-q / p}}\right)^{1 / q}<\infty\right\} .
$$

The space $\ell_{p, q}$ is the Lorentz sequence space $d(w, q)$ with $w(k)=k^{q / p-1}$. 
We apply the above results to these particular spaces. By Theorem 1.1(a), for $n \leq q$, (2) holds for $E=\ell_{p, q}$ if and only if $\alpha \in\left(\ell_{p / n, q / n}\right)^{*}$.

If $n \geq p$, then since $\ell_{p, q} \hookrightarrow \ell_{n},(2)$ holds if and only if $\alpha \in \ell_{\infty}$.

Finally, for $q<n<p$ we can apply Theorem 1.1(b). Since $w$ is regular and $\tilde{w}(k)=(k w(k))^{n / q} / k=k^{n / p-1}$ is a decreasing sequence, Remark 3.3 gives that (2) holds if and only if $\alpha \in d(\tilde{w}, 1)^{*}=\left(\ell_{p / n, 1}\right)^{*}$.

It is easy to check that the sequence $\left(k^{q / p-1}\right)_{k}$ is $r$-regular if and only if $r<p /(p-q)$. Therefore, for any $r<n /(n-q)$ we can find $p>n$ such that $r<p /(p-q)$. In this case, the sequence associated to $\ell_{p, q}$ is $r$-regular but (2) does not hold for some $\alpha \in \ell_{\infty}$.

4. Duals of Lorentz spaces. We now prove Theorem 1.2. We have seen in Section 3 that using diagonal $n$-linear forms can sometimes be helpful. In the same spirit, an operator $D \in \mathcal{L}(E ; F)$ between Köthe sequence spaces is called diagonal if there exists a sequence $\sigma$ such that $D(x)=(\sigma(k) x(k))_{k=1}^{\infty}$; in this case we write $D=D_{\sigma}$. Some relationship between diagonal operators and diagonal $n$-linear forms is shown in the following lemma, which we will need later.

Lemma 4.1. Let $E$ be a symmetric Köthe sequence space and $T_{\alpha}: E \times$ $\cdots \times E \rightarrow \mathbb{C}$ a diagonal n-linear form. Let $D_{\sigma}: E \rightarrow \ell_{n}$ be the diagonal operator associated to $\sigma=\alpha^{1 / n}$ (coordinatewise). Then $T_{\alpha}$ is continuous if and only if $D_{\sigma}$ is continuous, and

$$
\left\|T_{\alpha}\right\|=\left\|D_{\sigma}\right\|^{n} .
$$

Proof. If $P_{\alpha}$ is the $n$-homogeneous polynomial associated to $T_{\alpha}$, then by Remark 3.2, we have $\left\|T_{\alpha}\right\|=\left\|P_{\alpha}\right\| \leq\left\|D_{\sigma}\right\|^{n}$.

On the other hand, if $|\lambda(k)|=1$ for all $j$, then $\left\|(\lambda(k) x(k))_{k}\right\|_{E}=\|x\|_{E}$ and

$$
\left\|T_{\alpha}\right\| \geq \sup _{\substack{\|x\|_{E} \leq 1 \\ \alpha(k) x(k)^{n} \geq 0}}\left|\sum_{k=1}^{\infty} \alpha(k) x(k)^{n}\right|=\sup _{\|x\|_{E} \leq 1} \sum_{k=1}^{\infty}|\alpha(k)||x(k)|^{n}=\left\|D_{\sigma}\right\|^{n} .
$$

Now we are ready to prove our theorem for duals of Lorentz spaces.

Proof of Theorem 1.2. Part (a) follows from Remark 2.1 and the fact that $d(w, p)^{*}$ is $n$-concave if and only if $d(w, p)$ is $n^{\prime}$-convex, and this happens if and only if $1 \leq n^{\prime} \leq p$. In this case the $n$-concavity constant $\mathbf{M}_{(n)}\left(d(w, p)^{*}\right)$ is 1 . Since the norm of a diagonal multilinear form coincides with the norm of its associated polynomial, the best constant is $\|\alpha\|_{\infty}$.

To get (b), take $\alpha \in \ell_{\infty}$ and $\sigma=\alpha^{1 / n}$. If $D_{\sigma}: d(w, p)^{*} \rightarrow \ell_{n}$ is the diagonal operator associated to $\sigma$ and $D_{\sigma}^{\prime}: \ell_{n^{\prime}} \rightarrow d(w, p)$ is the adjoint 
operator, we want to show that

$$
\left\|D_{\sigma}^{\prime}\right\|=\|\alpha\|_{d\left(w^{\frac{n^{\prime}}{n^{\prime}-p}}, \frac{p^{\prime}}{p^{\prime}-n}\right)}^{1 / n} .
$$

If this is the case, then by Lemma 4.1,

$$
\|\alpha\|_{d\left(w^{\frac{n^{\prime}}{n^{\prime}-p}}, \frac{p^{\prime}}{p^{\prime}-n}\right)}=\left\|D_{\sigma}^{\prime}\right\|^{n}=\left\|D_{\sigma}\right\|^{n}=\left\|T_{\alpha}\right\|
$$

and for all $x_{1}, \ldots, x_{n} \in d(w, p)^{*}$,

$$
\left|\sum_{k} \alpha(k) x_{1}(k) \cdots x_{n}(k)\right| \leq\|\alpha\|_{d\left(w^{\frac{n^{\prime}}{n^{\prime}-p}}, \frac{p^{\prime}}{p^{\prime}-n}\right)}\left\|x_{1}\right\| \cdots\left\|x_{n}\right\| .
$$

Hence, (2) holds if and only if $\alpha \in d\left(w^{\frac{n^{\prime}}{n^{\prime}-p}}, \frac{p^{\prime}}{p^{\prime}-n}\right)$, and the best constant is the norm of $\alpha$ in this space.

Let us now show that (8) holds. First,

$$
\left\|D_{\sigma}^{\prime}(x)\right\|=\left\|(\sigma(k) x(k))_{k}\right\|_{d(w, p)}=\sup _{\pi \in \Sigma_{\mathbb{N}}}\left(\sum_{k}\left|\alpha(\pi(k))^{1 / n} x(\pi(k))\right|^{p} w(k)\right)^{1 / p} .
$$

Using Hölder's inequality with exponents $n^{\prime} / p$ and $n^{\prime} /\left(n^{\prime}-p\right)$ we obtain, for each $\pi \in \Sigma_{\mathbb{N}}$,

$$
\begin{aligned}
\left(\sum_{k}\left|\alpha(\pi(k))^{1 / n} x(\pi(k))\right|^{p} w(k)\right)^{1 / p} & \\
& \leq\left(\sum_{k}|x(\pi(k))|^{n^{\prime}}\right)^{1 / n^{\prime}}\left(\sum_{k}|\alpha(\pi(k))|^{\frac{p^{\prime}}{p^{\prime}-n}} w(k)^{\frac{n^{\prime}}{n^{\prime}-p}}\right)^{\frac{n^{\prime}-p}{n^{\prime} p}} \\
& \leq\|x\|_{\ell_{n^{\prime}}}\left(\sum_{k} \alpha^{\star}(k)^{\frac{p^{\prime}}{p^{\prime}-n}} w(k)^{\frac{n^{\prime}}{n^{\prime}-p}}\right)^{\frac{p^{\prime}-n}{p^{\prime} n}} .
\end{aligned}
$$

Hence

$$
\left\|D_{\sigma}^{\prime}\right\| \leq\|\alpha\|_{d\left(w^{\frac{n^{\prime}}{n^{\prime}-p}}, \frac{p^{\prime}}{p^{\prime}-n}\right)}^{1 / n} .
$$

Let us see now that this value is attained. Since all the spaces involved are symmetric we can assume that $\alpha=\alpha^{\star}$. Let

$$
x_{N}(k)=\frac{\alpha(k)^{\frac{p}{\left(n^{\prime}-p\right) n}} w(k)^{\frac{1}{n^{\prime}-p}}}{\left(\sum_{i=1}^{N} \alpha(i)^{\frac{p^{\prime}}{p^{\prime}-n}} w(i)^{\frac{n^{\prime}}{n^{\prime}-p}}\right)^{1 / n^{\prime}}}
$$

for $k=1, \ldots, N$. It is easily seen that $\left\|\left(x_{N}(k)\right)_{k=1}^{N}\right\|_{\ell_{n^{\prime}}}=1$ and 


$$
\begin{aligned}
\left\|D_{\sigma}^{\prime}\left(x_{N}\right)\right\|_{d(w, p)} & =\left(\sum_{k=1}^{N} \alpha(k)^{\frac{p^{\prime}}{p^{\prime}-n}} w(k)^{\frac{n^{\prime}}{n^{\prime}-p}}\right)^{1 / p-1 / n^{\prime}} \\
& =\left\|\sum_{k=1}^{N} \alpha(k) e_{j}\right\|_{d\left(w^{\frac{n^{\prime}}{n^{\prime}-p}}, \frac{p^{\prime}}{p^{\prime}-n}\right)}^{1 / n}
\end{aligned}
$$

Therefore

$$
\left\|\sum_{k=1}^{N} \alpha(k) e_{j}\right\|_{d\left(w^{\frac{n^{\prime}}{n^{\prime}-p}}, \frac{p^{\prime}}{p^{\prime}-n}\right)}^{1 / n} \leq\left\|D_{\sigma}^{\prime}\right\|
$$

for all $N$ and the result follows.

Statement (c) follows similarly.

5. A general approach. We have seen in Sections 3 and 4 that considering diagonal $n$-linear forms helps in proving Hölder-type inequalities. In fact, if in (2) we take the supremum over $\left\|x_{i}\right\|_{E} \leq 1, i=1, \ldots, n$, then we find that the best constant in (2) is precisely $\left\|T_{\alpha}\right\|$. We see that our problem is closely related to determining the norm of diagonal $n$-linear forms. This sits very much in the philosophy of considering norms of diagonal multilinear forms in different ideals presented in $[5,6]$ and motivates us to broaden our framework.

Following [18] for the linear case and [6] for the multilinear case, if $\mathfrak{A}$ is a Banach ideal of multilinear mappings we consider, for each $n \in \mathbb{N}$, the space

$$
\ell_{n}(\mathfrak{A}, E):=\left\{\alpha \in \ell_{\infty}: T_{\alpha} \in \mathfrak{A}\left({ }^{n} E\right)\right\} .
$$

With the norm $\|\alpha\|_{\ell_{n}(\mathfrak{A}, E)}=\left\|T_{\alpha}\right\|_{\mathfrak{A}\left({ }^{n} E\right)}$, it is a symmetric Köthe sequence space whenever $E$ is.

If $\mathcal{L}$ denotes the ideal of all multilinear forms, then (1) can be rewritten as

$$
\ell_{n}\left(\mathcal{L}, \ell_{p}\right) \stackrel{1}{=} \begin{cases}\ell_{\infty} & \text { if } 1 \leq p \leq n, \\ \ell_{p /(p-n)} & \text { if } n<p<\infty\end{cases}
$$

and Theorems 1.1 and 1.2 can be summarized as

$$
\begin{gathered}
\ell_{n}(\mathcal{L}, d(w, p)) \stackrel{1}{=} \begin{cases}d(w, p / n)^{*} & \text { if } n \leq p, \\
m_{\Psi} & \text { if } n>p,\end{cases} \\
\ell_{n}\left(\mathcal{L}, d(w, p)^{*}\right) \stackrel{1}{=} \begin{cases}\ell_{\infty} & \text { if } n^{\prime} \leq p, \\
d\left(w^{\frac{n^{\prime}}{n^{\prime}-p}}, \frac{p^{\prime}}{p^{\prime}-n}\right) & \text { if } n^{\prime}>p>1, \\
d\left(w^{n}, 1\right) & \text { if } p=1,\end{cases}
\end{gathered}
$$

where $\Psi(N)=\left(\sum_{j=1}^{N} w(j)\right)^{n / p}$. If $n>p$ and $w$ is $n /(n-p)$-regular, then $\ell_{n}(\mathcal{L}, d(w, p))=\ell_{\infty}$.

Our aim in this section is to obtain descriptions of $\ell_{n}(\mathfrak{A}, d(w, p))$ and $\ell_{n}\left(\mathfrak{A}, d(w, p)^{*}\right)$ for ideals $\mathfrak{A}$ other than $\mathcal{L}$. We will make use of some general 
facts. If $E$ is a Köthe sequence space, we consider the mapping $\Phi_{N}: E_{N} \times$ $\cdots \times E_{N} \rightarrow \mathbb{C}$ given by

$$
\Phi_{N}\left(x_{1}, \ldots, x_{n}\right)=\sum_{k=1}^{N} x_{1}(k) \cdots x_{n}(k) .
$$

Clearly $\left\|\Phi_{N}\right\|_{\mathfrak{A}\left({ }^{n} E\right)}=\lambda_{\ell_{n}(\mathfrak{A}, E)}(N)$.

If $F$ and $G$ are symmetric Köthe sequence spaces so that $F \hookrightarrow G$ then, by the closed graph theorem,

$$
\lambda_{G}(N) \prec \lambda_{F}(N) .
$$

A weak converse of this fact can be obtained under certain assumptions. We first need a lemma.

Lemma 5.1. Let $F$ and $G$ symmetric Köthe sequence spaces and suppose there exists $\alpha>0$ such that $\lambda_{G}(N) \prec \lambda_{F}(N)^{\alpha}$. Then

$$
\left(\frac{1}{k^{\varepsilon} \lambda_{F}(k)^{\alpha}}\right)_{k \in \mathbb{N}} \in G \quad \text { for all } \varepsilon>0 .
$$

Proof. For each $m \in \mathbb{N} \cup\{0\}$, we define

$$
\mathbb{N}_{m}=\left\{k \in \mathbb{N}: 2^{m} \leq k<2^{m+1}\right\} \quad \text { and } \quad x_{m}=\sum_{k \in \mathbb{N}_{m}} e(k) .
$$

Since $G$ is symmetric, $\left\|x_{m}\right\|_{G}=\lambda_{G}\left(2^{m}\right) \prec \lambda_{F}\left(2^{m}\right)^{\alpha}$. Hence,

$$
\sum_{m} \frac{1}{2^{m \varepsilon} \lambda_{F}\left(2^{m}\right)^{\alpha}} x_{m} \in G \text {. }
$$

Now, for $k \in \mathbb{N}_{m}$, we have $1 / k \leq 1 / 2^{m}$ and $1 / \lambda_{F}(k) \leq 1 / \lambda_{F}\left(2^{m}\right)$, and the result follows.

Proposition 5.2. Let $F$ and $G$ be symmetric Köthe sequence spaces for which there exists $0<\varepsilon<1$ such that $\lambda_{G}(N) \prec \lambda_{F}(N)^{1-\varepsilon}$. If $N^{\delta} \prec \lambda_{F}(N)$ for some $\delta>0$, then $F \hookrightarrow G$.

Proof. Let $x \in F$. We can assume that $x(k)=x^{\star}(k)$ is decreasing. Then

$$
x(k) \lambda_{F}(k) \leq\left\|\sum_{j=1}^{k} x(j) e_{j}\right\|_{F} \leq\|x\|_{F} .
$$

Now, $\lambda_{F}(k)=\lambda_{F}(k)^{\varepsilon} \lambda_{F}(k)^{1-\varepsilon} \succ k^{\varepsilon \delta} \lambda_{F}(k)^{1-\varepsilon}$. Hence

By Lemma 5.1, $x \in G$.

$$
x(k) \prec \frac{\|x\|_{F}}{k^{\varepsilon \delta} \lambda_{F}(k)^{1-\varepsilon}} .
$$

Note that the additional condition on the sequence space $F$ is automatically satisfied whenever $F$ or $G$ have non-trivial concavity. The previous results can be reformulated to obtain information on the space $\ell_{n}(\mathfrak{A}, E)$. 
Corollary 5.3. Let $E, F$ and $G$ be symmetric Köthe sequence spaces and $\mathfrak{A}$ be a Banach ideal of multilinear forms.

(a) If $F \hookrightarrow \ell_{n}(\mathfrak{A}, E) \hookrightarrow G$, then $\lambda_{G}(N) \prec\left\|\Phi_{N}\right\|_{\mathfrak{A}\left({ }^{n} E\right)} \prec \lambda_{F}(N)$.

(b) If there exists $\varepsilon>0$ such that $\left\|\Phi_{N}\right\|_{\mathfrak{A}\left({ }^{(n} E_{N}\right)} \prec \lambda_{F}(N)^{1-\varepsilon}$ and $F$ has non-trivial concavity, then $F \hookrightarrow \ell_{n}(\mathfrak{A}, E)$.

(c) If there exists $\varepsilon>0$ such that $\lambda_{G}(N)^{1+\varepsilon} \prec\left\|\Phi_{N}\right\|_{\mathfrak{A}^{\left({ }^{n} E_{N}\right)}}$ and $G$ has non-trivial concavity, then $\ell_{n}(\mathfrak{A}, E) \hookrightarrow G$.

If $\mathfrak{A}$ is a normed ideal of $n$-linear forms, the maximal hull $\mathfrak{A}^{\max }$ of $\mathfrak{A}$ is defined as the class of all $n$-linear forms $T$ such that

$$
\begin{array}{r}
\|T\|_{\mathfrak{A}^{\max }\left(E_{1}, \ldots, E_{n}\right)}:=\sup \left\{\left\|\left.T\right|_{M_{1} \times \cdots \times M_{n}}\right\|_{\mathfrak{A}\left(M_{1}, \ldots, M_{n}\right)}:\right. \\
\left.M_{i} \subset E_{i}, \operatorname{dim} M_{i}<\infty\right\}
\end{array}
$$

is finite. $\mathfrak{A}^{\max }$ is always complete and it is the largest ideal whose norm coincides with $\|\cdot\|_{\mathfrak{A}}$ in finite-dimensional spaces. A normed ideal $\mathfrak{A}$ is called maximal if $\left(\mathfrak{A},\|\cdot\|_{\mathfrak{A}}\right)=\left(\mathfrak{A}^{\max },\|\cdot\|_{\mathfrak{A}^{\max }}\right)$. Maximal ideals are those whose norms are uniquely determined by finite-dimensional subspaces.

It is a well known fact that the space of $n$-linear forms on a finitedimensional space $M$ can be identified with the $n$-fold tensor product $\bigotimes^{n} M^{*}$ by identifying each tensor $\gamma_{1} \otimes \cdots \otimes \gamma_{n}$ with the mapping $\left(x_{1}, \ldots, x_{n}\right) \rightsquigarrow$ $\gamma_{1}\left(x_{1}\right) \cdots \gamma_{n}\left(x_{n}\right)$. Then the ideal norm induces a tensor norm $\eta$ on $\bigotimes^{n} M^{*}$ (the tensor product with this norm is denoted by $\bigotimes_{\eta}^{n} M^{*}$ ). By a standard procedure the norm $\eta$ can be extended from tensor norms in the class of finite-dimensional normed spaces to the class of all normed spaces. In this case, the tensor norm $\eta$ and the ideal $\mathfrak{A}$ are said to be associated. A detailed study of the subject and presentation of the procedure can be found in $[8$, 10-13].

Given a normed ideal $\mathfrak{A}$ associated to the finitely generated tensor norm $\alpha$, its adjoint ideal $\mathfrak{A}^{*}$ is defined by

$$
\mathfrak{A}^{*}\left({ }^{n} E\right):=\left(\bigotimes_{\eta}^{n} E\right)^{*} .
$$

The adjoint ideal is called the dual ideal in [10]. The tensor norm associated to $\mathfrak{A}^{*}$ is denoted by $\eta^{*}$. We also have the representation theorem $[13$, Section $3.2]$ (see also [10, Section 4])

$$
\mathfrak{A}^{\max }\left({ }^{n} E\right)=\left(\bigotimes_{\eta^{*}}^{n} E\right)^{*} .
$$

In particular, this shows that the adjoint ideal $\mathfrak{A}^{*}$ is maximal.

For a maximal ideal $\mathfrak{A}$, the space $\ell_{n}(\mathfrak{A}, E)$ coincides isometrically with $\ell_{n}\left(\mathfrak{A}, E^{\times \times}\right)$. This is a consequence of the following lemma.

Lemma 5.4. Let $E$ be a symmetric Köthe sequence space and $\mathfrak{A}$ a maximal Banach ideal of multilinear forms. Let $T: E \times \cdots \times E \rightarrow \mathbb{C}$ be a diagonal $n$-linear form and suppose there exists $C>0$ such that, for every $N \in \mathbb{N}$, the 
restriction $T^{N}$ to $E_{N} \times \cdots \times E_{N}$ satisfies $\left\|T^{N}\right\|_{\mathfrak{A}\left({ }^{n} E_{N}\right)} \leq C$. Then $T \in \mathfrak{A}\left({ }^{n} E\right)$ and $\|T\|_{\mathfrak{A}\left({ }^{n} E\right)} \leq C$.

Proof. Since $\mathfrak{A}$ is maximal, there exists a finitely generated tensor norm $\nu$ such that $\left(\bigotimes_{\nu}^{n} E\right)^{*}=\mathfrak{A}\left({ }^{n} E\right)$. Also, $E$ being a symmetric space, both the inclusion $i_{N}: E_{N} \hookrightarrow E$ and the projection $\pi_{N}: E \rightarrow E_{N}$ have norm 1 . Now, the hypotheses mean that the sequence $\left(T^{N}\right)_{N}$ is contained in the ball $C B_{\mathfrak{A}\left({ }^{n} E\right)}$, which is a weak-star compact set (by the weak-star topology we mean the topology on $\mathfrak{A}\left({ }^{n} E\right)$ considered as the dual of $\left.\bigotimes_{\nu}^{n} E\right)$. Therefore, it has a weak-star accumulation point $S$ in $C B_{\mathfrak{A}\left({ }^{n} E\right)}$. But, since $T$ is diagonal, the truncations $T^{N}$ converge pointwise to $T$, so $S$ must coincide with $T$ and consequently $T$ belongs to $C B_{\mathfrak{A}\left({ }^{n} E\right)}$, which ends the proof.

The previous lemma also holds if $E$ is a Banach space with a 1-unconditional basis and $T$ is an arbitrary (not necessarily diagonal) multilinear form. In this case, we can apply the $n$-linear version of the Density Lemma $[8,13.4]$, considering $E_{0}$ (the subspace of $E$ spanned by the canonical basis) as the dense subspace of $E$. Also, if $E$ has an unconditional basis with unconditional constant $K$, we obtain the conclusion with $\|T\|_{\mathfrak{A}\left({ }^{n} E\right)} \leq K C$.

Proposition 5.5. Let $E$ be a symmetric Köthe sequence space and $\mathfrak{A}$ a maximal Banach ideal of multilinear forms. Then

$$
\ell_{n}(\mathfrak{A}, E) \stackrel{1}{=} \ell_{n}\left(\mathfrak{A}, E^{\times \times}\right) .
$$

Proof. Since $E$ is contained in $E^{\times \times}$with a norm one inclusion, it is immediate that $\ell_{n}\left(\mathfrak{A}, E^{\times \times}\right) \subset \ell_{n}(\mathfrak{A}, E)$ (with norm one inclusion).

Conversely, let $\alpha \in \ell_{n}(\mathfrak{A}, E)$. For each $N,\left\|T_{\alpha}^{N}\right\|_{\mathfrak{A}\left({ }^{n} E_{N}\right)} \leq\left\|T_{\alpha}\right\|_{\mathfrak{A}\left({ }^{n} E\right)}$. Since $E_{N} \stackrel{1}{=}\left(E_{N}\right)^{\times \times} \stackrel{1}{=}\left(E^{\times \times}\right)_{N}$, we have $\left\|T_{\alpha}^{N}\right\|_{\mathfrak{A}\left({ }^{n}\left(E^{\times \times}\right)_{N}\right)} \leq\left\|T_{\alpha}\right\|_{\mathfrak{A}\left({ }^{n} E\right)}$. By Lemma 5.4, $T_{\alpha}$ belongs to $\mathfrak{A}\left({ }^{n} E^{\times \times}\right)$and $\left\|T_{\alpha}\right\|_{\mathfrak{A}\left({ }^{n} E^{\times \times}\right)} \leq\left\|T_{\alpha}\right\|_{\mathfrak{A}\left({ }^{n} E\right)}$.

The ideal $\mathcal{L}$ of all multilinear forms is obviously maximal; then by Theorem $1.2(\mathrm{c})$ we have the following reformulation of [22, Theorem 2.5]:

$$
\ell_{n}\left(\mathcal{L}, d_{*}(w, 1)\right) \stackrel{1}{=} d\left(w^{n}, 1\right) .
$$

Let us recall the trace duality between $\mathfrak{A}^{*}\left({ }^{n} E_{N}^{\times}\right)$and $\mathfrak{A}\left({ }^{n} E_{N}\right)$. Suppose $T \in \mathfrak{A}^{*}\left({ }^{n} E_{N}^{\times}\right)$can be written as a finite sum of the form

$$
T\left(\gamma_{1}, \ldots, \gamma_{n}\right)=\sum_{j} \gamma_{1}\left(x_{1}^{j}\right) \cdots \gamma_{n}\left(x_{n}^{j}\right)
$$

and $S \in \mathfrak{A}\left({ }^{n} E_{N}\right)$ is of the form

$$
S\left(x_{1}, \ldots, x_{n}\right)=\sum_{i} \gamma_{1}^{i}\left(x_{1}\right) \cdots \gamma_{n}^{i}\left(x_{n}\right) .
$$


Then the duality is given by

$$
\begin{aligned}
\langle T, S\rangle & =\sum_{i, j} \gamma_{1}^{i}\left(x_{1}^{j}\right) \cdots \gamma_{n}^{i}\left(x_{n}^{j}\right) \\
& =\sum_{i} T\left(\gamma_{1}^{i}, \ldots, \gamma_{n}^{i}\right)=\sum_{j} S\left(x_{1}^{j}, \ldots, x_{n}^{j}\right) .
\end{aligned}
$$

The following finite-dimensional identifications are easy to check. These will enable us to prove a duality result in the proposition below.

$$
\begin{aligned}
& \ell_{n}\left(\mathfrak{A}, E_{N}\right) \stackrel{1}{=}\left[\ell_{n}(\mathfrak{A}, E)\right]_{N}, \\
& \mathfrak{A}\left({ }^{n} E_{N}\right)^{*} \stackrel{1}{=} \mathfrak{A}^{*}\left({ }^{n} E_{N}^{\times}\right) \stackrel{1}{=} \mathfrak{A}^{*}\left({ }^{n} E_{N}^{*}\right), \\
& \ell_{n}(\mathfrak{A}, E)_{N}^{\times} \stackrel{1}{=} \ell_{n}\left(\mathfrak{A}, E_{N}\right)^{\times} \stackrel{1}{=} \ell_{n}\left(\mathfrak{A}^{*}, E_{N}^{\times}\right) \stackrel{1}{=} \ell_{n}\left(\mathfrak{A}^{*}, E^{\times}\right)_{N} .
\end{aligned}
$$

Proposition 5.6. Let $E$ be a symmetric Köthe sequence space and $\mathfrak{A} a$ Banach ideal of multilinear forms. Then

$$
\ell_{n}(\mathfrak{A}, E)^{\times} \stackrel{1}{=} \ell_{n}\left(\mathfrak{A}^{*}, E^{\times}\right) .
$$

Proof. Take first $\alpha \in \ell_{n}(\mathfrak{A}, E)^{\times}$; then the associated $n$-linear form $T_{\alpha}$ is defined on the space of finite sequences in $E^{\times}$. Moreover, using (12), we have

$$
\begin{aligned}
\left\|\left.T_{\alpha}\right|_{E_{N}^{\times} \times \cdots \times E_{N}^{\times}}\right\|_{\mathfrak{A}^{*}\left({ }^{n} E_{N}^{\times}\right)} & =\left\|\pi_{N}(\alpha)\right\|_{\ell_{n}\left(\mathfrak{A}^{*}, E_{N}^{\times}\right)} \\
& =\left\|\pi_{N}(\alpha)\right\|_{\ell_{n}(\mathfrak{A}, E)_{N}^{\times}} \leq\|\alpha\|_{\ell_{n}(\mathfrak{A}, E)^{\times}} .
\end{aligned}
$$

By Lemma 5.4, $\alpha$ belongs to $\ell_{n}\left(\mathfrak{A}^{*}, E^{\times}\right)$and $\|\alpha\|_{\ell_{n}\left(\mathfrak{A}^{*}, E^{\times}\right)}=\left\|T_{\alpha}\right\|_{\mathfrak{A}^{*}\left({ }^{n} E^{\times}\right)} \leq$ $\|\alpha\|_{\ell_{n}(\mathfrak{A}, E)^{\times}}$.

Take now $\alpha \in \ell_{n}\left(\mathfrak{A}^{*}, E^{\times}\right)$and a norm one $\beta \in \ell_{n}(\mathfrak{A}, E)$. For each $j$, let $\tilde{\beta}(j)$ be such that $\alpha(j) \tilde{\beta}(j)=|\alpha(j) \beta(j)|$. Then, by symmetry and (9),

$$
\begin{aligned}
& \sum_{j=1}^{N}|\alpha(j) \beta(j)|=\sum_{j=1}^{N} \alpha(j) \tilde{\beta}(j)=\left\langle T_{\pi_{N}(\alpha)}, T_{\pi_{N}(\tilde{\beta})}\right\rangle_{\mathfrak{A}^{*}\left({ }^{n} E_{N}^{\times}\right), \mathfrak{A}\left({ }^{n} E_{N}\right)} \\
& \leq\left\|T_{\alpha}\right\|_{\mathfrak{R}^{*}\left({ }^{n} E^{\times}\right)}\left\|T_{\tilde{\beta}}\right\|_{\mathfrak{A}\left({ }^{n} E\right)}=\left\|T_{\alpha}\right\|_{\mathfrak{A}^{*}\left({ }^{n} E^{\times}\right)}\left\|T_{\beta}\right\|_{\mathfrak{A}\left({ }^{n} E\right)}=\|\alpha\|_{\ell_{n}\left(\mathfrak{A}^{*}, E^{\times}\right)}
\end{aligned}
$$

This completes the proof.

By applying Proposition 5.6 to the adjoint ideal and to the Köthe dual of $E$, and Proposition 5.5, we get

$$
\ell_{n}\left(\mathfrak{A}^{*}, E^{\times}\right)^{\times}=\ell_{n}\left(\mathfrak{A}^{* *}, E^{\times \times}\right)=\ell_{n}\left(\mathfrak{A}^{\max }, E^{\times \times}\right)=\ell_{n}\left(\mathfrak{A}^{\max }, E\right)
$$

isometrically. Therefore, if $\mathfrak{A}$ is maximal we immediately have

$$
\ell_{n}(\mathfrak{A}, E) \stackrel{1}{=} \ell_{n}\left(\mathfrak{A}^{*}, E^{\times}\right)^{\times} .
$$

In view of Proposition 5.6 we can use Theorems 1.1 and 1.2 to get results on ideals other than $\mathcal{L}$. Let us recall that $T \in \mathcal{L}\left({ }^{n} E\right)$ is called nuclear if 
there are sequences $\left(\gamma_{1, k}\right)_{k}, \ldots,\left(\gamma_{n, k}\right)_{k}$ in $E^{*}$ with $\left\|\gamma_{i, k}\right\| \leq 1$ for all $k$ and $i=1, \ldots, n$ and there is $(\lambda(k))_{k} \in \ell_{1}$ so that for every $x_{1}, \ldots, x_{n} \in E$,

$$
T\left(x_{1}, \ldots, x_{n}\right)=\sum_{k} \lambda(k) \cdot \gamma_{1, k}\left(x_{1}\right) \cdots \gamma_{n, k}\left(x_{n}\right) .
$$

We denote by $\mathcal{N}$ the ideal of nuclear forms. The nuclear norm is defined as the infimum of $\sum_{k}|\lambda(k)|\left\|\gamma_{1, k}\right\| \cdots\left\|\gamma_{n, k}\right\|$ over all possible representations.

A mapping $T \in \mathcal{L}\left({ }^{n} E\right)$ is called integral if there exists a positive BorelRadon measure $\mu$ on $B_{E^{*}} \times \cdots \times B_{E^{*}}$ (with the weak* topologies) such that

$$
T\left(x_{1}, \ldots, x_{n}\right)=\int_{B_{E^{*}} \times \times B_{E^{*}}} \gamma_{1}\left(x_{1}\right) \cdots \gamma_{n}\left(x_{n}\right) d \mu\left(\gamma_{1}, \ldots, \gamma_{n}\right)
$$

for all $x_{1}, \ldots, x_{n} \in X$ (see $[8,4.5]$ and [1]). The ideal of integral multilinear forms is denoted by $\mathcal{I}$. It is well known that $\mathcal{L}^{*}=\mathcal{I}$. We then have

$$
\begin{gathered}
\ell_{n}(\mathcal{I}, d(w, p)) \stackrel{1}{=} \begin{cases}d\left(w^{n}, 1\right)^{*} & \text { if } p=1, \\
d\left(w^{\frac{n^{\prime}}{n^{\prime}}-p}, \frac{p^{\prime}}{p^{\prime}-n}\right)^{*} & \text { if } 1<p<n^{\prime}, \\
\ell_{1} & \text { if } n^{\prime} \leq p,\end{cases} \\
\ell_{n}\left(\mathcal{I}, d(w, p)^{*}\right) \stackrel{1}{=} \begin{cases}m_{\Psi}^{\times}=\left(m_{\Psi}^{0}\right)^{*} & \text { if } 1 \leq p<n, \\
d(w, p / n) & \text { if } n \leq p .\end{cases}
\end{gathered}
$$

Here $m_{\Psi}^{0}$ denotes the subspace of order continuous elements of $m_{\Psi}$, and satisfies $\left(m_{\Psi}^{0}\right)^{* *}=m_{\Psi}$ (see [16]). The equality $m_{\Psi}^{\times}=\left(m_{\Psi}^{0}\right)^{*}$ follows from the proof of [16, Theorem 3.4].

Whenever a space $E$ is reflexive or has a separable dual, the nuclear and integral mappings on $E$ coincide. Therefore, for $1<p<\infty, \ell_{n}(\mathcal{I}, d(w, p))=$ $\ell_{n}(\mathcal{N}, d(w, p))$ and $\ell_{n}\left(\mathcal{I}, d(w, p)^{*}\right)=\ell_{n}\left(\mathcal{N}, d(w, p)^{*}\right)$. Also, $\ell_{n}\left(\mathcal{N}, d_{*}(w, 1)\right)=$ $\ell_{n}\left(\mathcal{I}, d_{*}(w, 1)\right)=\ell_{n}\left(\mathcal{I}, d^{*}(w, 1)\right)$ (the last equality follows from Proposition 5.5).

By Remark 3.3, for $p<n, \ell_{n}\left(\mathcal{I}, d(w, p)^{*}\right)$ can be identified isomorphically with $d(\bar{w}, 1)^{* *}$ for some $\bar{w}$. Moreover, if $p<n$ and $w$ is $n /(n-p)$-regular, then $\ell_{n}\left(\mathcal{I}, d(w, p)^{*}\right)=\ell_{1}$ by Theorem 1.1.

REMARK 5.7. We have already mentioned that $\left\|\Phi_{N}\right\|_{\mathfrak{A}\left({ }^{n} E\right)}=\lambda_{\ell_{n}(\mathfrak{A}, E)}(N)$ always holds. Therefore, all the previous results immediately give estimates for the usual and the nuclear norms of $\Phi_{N}$ (the nuclear and integral norms of $\Phi_{N}$ always coincide).

Moreover, these estimates have an immediate tensor counterpart, since $\left\|\Phi_{N}\right\|_{\mathcal{L}\left({ }^{n} E\right)}=\left\|\sum_{j=1}^{N} e_{j}^{\prime} \otimes \cdots \otimes e_{j}^{\prime}\right\|_{\bigotimes_{\varepsilon}^{n} E^{\prime}}$ and $\left\|\Phi_{N}\right\|_{\mathcal{N}\left({ }^{n} E\right)}=\| \sum_{j=1}^{N} e_{j}^{\prime} \otimes \cdots \otimes$ $e_{j}^{\prime} \|_{\otimes_{\pi}^{n} E^{\prime}}(\varepsilon$ and $\pi$ denote respectively the injective and projective tensor norms). 
Acknowledgements. We would like to thank Silvia Lassalle and Andreas Defant for helpful conversations and suggestions that improved the final shape of the paper. We would also like to thank the referee for her/his valuable suggestions.

Most of the work in this article was performed while the third-named author was visiting the Departments of Mathematics at Universidad de Buenos Aires and Universidad de San Andrés during the summer/winter of 2006. He wishes to thank all the people in and outside both Departments that made that such a delightful time.

The first-named author was partially supported by ANPCyT PICT 05 17-33042, UBACyT Grant X038 and ANPCyT PICT 06 00587. The secondnamed author was partially supported by ANPCyT PICT 05 17-33042. The third-named author was supported by the MEC Project MTM2008-03211 and partially by grants GV-AEST06/092 and UPV-PAID-00-06.

\section{References}

[1] R. Alencar, Multilinear mappings of nuclear and integral type, Proc. Amer. Math. Soc. 94 (1985), 33-38.

[2] G. D. Allen, Duals of Lorentz spaces, Pacific J. Math. 77 (1978), 287-291.

[3] C. Bennett and R. Sharpley, Interpolation of Operators, Academic Press, Orlando, 1988.

[4] Y. Benyamini, S. Lassalle, and J. G. Llavona, Homogeneous orthogonally additive polynomials on Banach lattices, Bull. London Math. Soc. 38 (2006), 459-469.

[5] D. Carando, V. Dimant, and P. Sevilla-Peris, Limit orders and multilinear forms on $l_{p}$ spaces, Publ. Res. Inst. Math. Sci. 42 (2006), 507-522.

[6] - , - - Ideals of multilinear forms - a limit order approach, Positivity 11 (2007), 589-607.

[7] Y. S. Choi and K. H. Han, Boundaries for algebras of holomorphic functions on Marcinkiewicz sequence spaces, J. Math. Anal. Appl. 323 (2006), 1116-1133.

[8] A. Defant and K. Floret, Tensor Norms and Operator Ideals, North-Holland Math. Stud. 176, North-Holland, Amsterdam, 1993.

[9] S. Dineen, Complex Analysis on Infinite-Dimensional Spaces, Springer Monogr. Math., Springer, London, 1999.

[10] K. Floret, Minimal ideals of n-homogeneous polynomials on Banach spaces, Results Math. 39 (2001), 201-217.

[11] -, On ideals of n-homogeneous polynomials on Banach spaces, in: Topological Algebras with Applications to Differential Geometry and Mathematical Physics (Athens, 1999), Univ. Athens, Athens, 2002, 19-38.

[12] K. Floret and D. García, On ideals of polynomials and multilinear mappings between Banach spaces, Arch. Math. (Basel) 81 (2003), 300-308.

[13] K. Floret and S. Hunfeld, Ultrastability of ideals of homogeneous polynomials and multilinear mappings on Banach spaces, Proc. Amer. Math. Soc. 130 (2002), 14251435.

[14] D. J. H. Garling, On symmetric sequence spaces, Proc. London Math. Soc. (3) 16 (1966), 85-106. 
[15] —, A class of reflexive symmetric BK-spaces, Canad. J. Math. 21 (1969), 602-608.

[16] A. Kamińska and H. J. Lee, $M$-ideal properties in Marcinkiewicz spaces, Comment. Math. Prace Mat., Tomus specialis in Honorem Juliani Musielak (2004), 123-144.

[17] - - - On uniqueness of extension of homogeneous polynomials, Houston J. Math. 32 (2006), 227-252.

[18] H. König, Diagonal and convolution operators as elements of operator ideals, Math. Ann. 218 (1975), 97-106.

[19] G. Krein, J. I. Petunin and E. M. Semenov, Interpolation of Linear Operators, Amer. Math. Soc., Providence, 1982.

[20] J. Lindenstrauss and L. Tzafriri, Classical Banach Spaces I. Sequence Spaces, Springer, Berlin, 1977.

[21] - - - Classical Banach Spaces II. Function Spaces, Springer, Berlin, 1979.

[22] M. L. Lourenço and L. Pellegrini, Interpolation by analytic functions on preduals of Lorentz sequence spaces, Glasgow Math. J. 48 (2006), 483-490.

[23] A. Pietsch, Ideals of multilinear functionals (designs of a theory), in: Proc. 2nd Int. Conf. on Operator Algebras, Ideals, and their Applications in Theoretical Physics (Leipzig, 1983), Teubner-Texte Math. 67, Teubner, Leipzig, 1984, 185-199.

[24] S. Reisner, A factorization theorem in Banach lattices and its application to Lorentz spaces, Ann. Inst. Fourier (Grenoble) 31 (1981), no. 1, 239-255.

[25] - On the duals of Lorentz function and sequence spaces, Indiana Univ. Math. J. 31 (1982), 65-72.

[26] W. L. C. Sargent, Some sequence spaces related to the $l^{p}$ spaces, J. London Math. Soc. 35 (1960), 161-171.

[27] K. Sundaresan, Geometry of spaces of homogeneous polynomials on Banach lattices, in: Applied Geometry and Discrete Mathematics, DIMACS Ser. Discrete Math. Theoret. Comput. Sci. 4, Amer. Math. Soc., Providence, RI, 1991, 571-586.

[28] N. Tomczak-Jaegermann, Banach-Mazur Distances and Finite-Dimensional Operator Ideals, Pitman Monogr. Surveys Pure Appl. Math. 38, Longman, Harlow, 1989.

Departamento de Matemática, Pab I

Facultad de Cs. Exactas y Naturales

Universidad de Buenos Aires

1428, Buenos Aires, Argentina

and

CONICET

E-mail: dcarando@dm.uba.ar

Instituto Universitario de Matemática Pura y Aplicada

ETSMRE - Universidad Politécnica de Valencia

Av. Blasco Ibáñez 21

46010 Valencia, Spain

E-mail: psevilla@mat.upv.es
Departamento de Matemática

Universidad de San Andrés

Vito Dumas 284

(B1644BID) Victoria, Buenos Aires, Argentina

and

CONICET

E-mail: vero@udesa.edu.ar

Received September 18, 2008

Revised version July 13, 2009 